
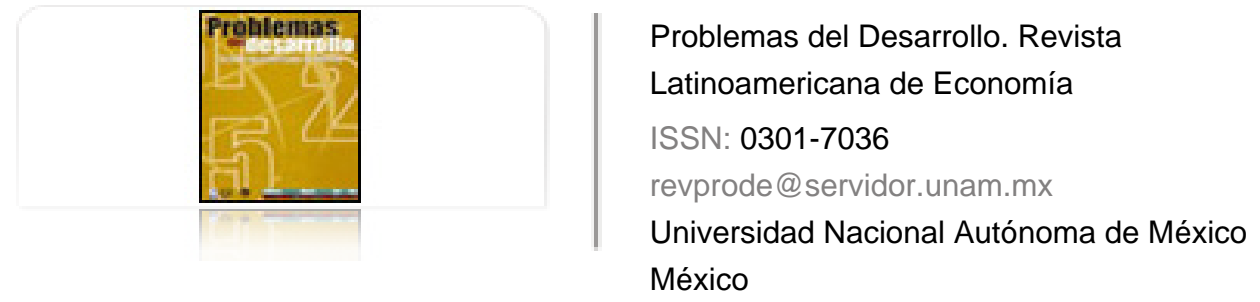

Panico, Carlo

Las políticas fiscales y monetarias en un ámbito comunitario

Problemas del Desarrollo. Revista Latinoamericana de Economía, vol. 42, núm. 164, enero-marzo, 2011, pp. 55-70

Universidad Nacional Autónoma de México

Distrito Federal, México

Disponible en: http://www.redalyc.org/articulo.oa?id=11819774004

Cómo citar el artículo

- Número completo

- Más información del artículo

Página de la revista en redalyc.org

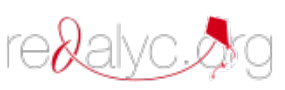

Sistema de Información Científica

Red de Revistas Científicas de América Latina, el Caribe, España y Portugal Proyecto académico sin fines de lucro, desarrollado bajo la iniciativa de acceso abierto 


\title{
LAS POLÍTICAS FISCALES Y MONETARIAS EN UN ÁMBITO COMUNITARIO
}

\author{
Carlo Panico*
}

Fecha de recepción: 4 de octubre de 2010. Fecha de aceptación: 10 de enero de 2011.

\begin{abstract}
RESUMEN
El ensayo examina las formas de coordinación de las políticas fiscales y monetarias en el área del euro. Hay una difundida insatisfacción en la literatura por el modo en que tal coordinación es organizada y muchos autores consideran que su carente funcionamiento contribuye a hacer crecer la economía del área menos de lo que se podría. El texto describe primero el marco institucional y de procedimiento de coordinación en el área del euro; después describe las evaluaciones de la literatura y las propuestas de reforma relacionadas. Finalmente, intenta delinear un marco institucional y de procedimiento que mejor refleje las evaluaciones presentes en la literatura que podrían contribuir a mejorar las potencialidades de crecimiento de la economía de esta área.
\end{abstract}

Palabras clave: Integración regional, desarrollo, coordinación de las políticas económicas, política monetaria y política fiscal.

\section{FISCAL AND MONETARY POLICIES IN A COMMUNITY ENVIRONMENT}

\section{Summary}

This paper examines the ways in which fiscal and monetary policies are coordinated in the euro zone. There is widespread dissatisfaction in the literature about the way this coordination is organized and many writers consider that its lack of functionality helps to make the area's economy grow less rapidly than it might. The paper first describes the institutional framework and the coordination procedure in the euro zone. It then goes on to describe the evaluations in the literature and the proposals for related reform. Finally, it tried to outline an institutional framework and procedure that would better reflect the assessments in the literature, and which could help to improve the potentialities for economic growth in this euro zone.

Key words: Regional integration, development, coordination of economic policies, monetary and fiscal policy.

1 Texto del seminario celebrado en la Facultad de Derecho de la Universidad de Roma, traducción de Miroslaba Lara Cancino y Miguel Ángel Isaac Lara Cancino. "La Sapienza”, 22 de octubre de 2007. Agradezco a los profesores Augusto Cerri y Gianluigi Galeotti por permitirme presentar anticipadamente los resultados de un trabajo de investigación que fueron después publicados en Panico y Vázquez Suárez (2008).

* Profesor-investigador de la Universidad Federico II de Nápoles. Correo electrónico: panico@ unina.it 


\section{LES POLITIQUES FISCALES ET MONÉTAIRES DANS UN ENVIRONNEMENT COMMUNAUTAIRE \\ Résumé}

L'essai examine les formes de coordination des politiques fiscales et monétaires dans la zone euro. Une insatisfaction est répandue dans la littérature pour la façon dont cette coordination est organisée et beaucoup d'auteurs considèrent que son fonctionnement défaillant contribue à ce que l'économie de la zone ne croisse pas autant qu'elle le pourrait. Le texte décrit d'abord le cadre institutionnel et de procédure de coordination dans la zone euro ; puis il décrit les évaluations de la littérature et les propositions de réformes qui y sont liées. Finalement, il essaie d'esquisser un cadre institutionnel et de procédure qui reflète mieux les évaluations présentes dans la littérature qui pourraient contribuer à améliorer les potentiels de croissance de l'économie de cette zone.

Mots clés : Intégration régionale, développement, coordination des politiques économiques, politique monétaire et politique fiscale

\section{AS POLÍTICAS FISCAIS E MONETÁRIAS NUM ÂMBITO COMUNITÁRIO Resumo}

O ensaio examina as formas de coordenação das políticas fiscais e monetárias na área do Euro. Há uma difundida insatisfação na literatura pelo modo no que tal coordenaçáo é organizada e muitos autores consideram que seu carente funcionamento contribui para que a economia da área cresça menos do que poderia. $\mathrm{O}$ texto descreve primeiramente o marco institucional e de procedimento de coordenaçáo na área do Euro; depois descreve as avaliaçóes da literatura e as propostas de reforma relacionadas. Finalmente, se tenta delinear um marco institucional e de procedimento que melhor reflita as avaliaçóes presentes na literatura que poderiam contribuir a melhorar as potencialidades de crescimento da economia desta área.

Palavras-chave: Integração regional, Desenvolvimento, coordenação de políticas econômicas, política monetária e política fiscal.

区域层面的财政政策与货币政策

摘要

本文探讨在欧元区的财政和货币政策的协调形式。许多有关资料明确显 示人们多这种协调的普遍不满。和许多作者认为, 其阻挡地区的增长潜 力。本文首先描述了欧元区的协调体制和程序框架, 然后介绍相关文章 的评估和改革建议。最后试图提出一个能够改善该地区的经济增长潜力 的体制和程序框架。

关键词：区域一体化，发展，经济政策协调，货币和财政政策。 


\section{INTRODUCCIÓN}

Se examinan aquí las formas de coordinación de las políticas fiscales y monetarias en el área del euro. Hay una difundida insatisfacción en la literatura por el modo en que tal coordinación es organizada y muchos autores consideran que su carente funcionamiento contribuye al crecimiento de la economía del área menos de lo que se podría.

En el decenio de 1999-2008, la economía del área del euro creció a una tasa real del $2.1 \%$ anual, frente a una capacidad de crecimiento en el mediano-largo plazo que el Banco Central Europeo ha estimado entre el 2 y el 2.5\%. Tal resultado se considera "débil" (ЕСв, 2008:66) y podría ser mejor si se toma en cuenta que en el mismo periodo el saldo comercial de la balanza de pagos registró excedentes persistentes que oscilaron entre el 1 y el 2\% del рів. Estos excedentes indujeron a los expertos a considerar que la política fiscal del área fuera excesivamente restrictiva a causa de la rigidez de las formas de coordinación existentes y que las limitaciones así impuestas a la demanda efectiva incidieron negativamente sobre las expectativas de los inversionistas, sobre sus gastos y, por lo tanto, sobre la productividad y el crecimiento del área.

La literatura señala que el actual marco institucional y de procedimiento de las actividades de coordinación incentiva a los gobiernos nacionales y al Banco Central Europeo a tener una postura no cooperativa. El debate, rico de análisis y propuestas, puede redirigirse hacia dos posiciones diversas: una que propone reformas de los procedimientos y una que considera necesario reformar las instituciones además de los procedimientos.

A estas posiciones corresponden visiones diversas del funcionamiento de la economía y significados diferentes atribuidos al término coordinación. Por un lado, hay autores (Buti, Eijffinger y Franco, 2002; Fatàs y Mihov, 2003; Beestma y Debrun, 2005; Calmfors, 2005), que hablan de la coordinación como una actividad encaminada a regular los comportamientos de los gobiernos nacionales y a garantizar la independencia al Banco Central (véase Fatàs y Mihov, 2003: 126). Por el otro, hay autores (Von Hagen y Mundschenk, 2003; Wyplosz, 1999, 2002; Pisani-Ferry, 2002), que describen la coordinación como un conjunto de actividades destinadas a identificar para toda el área del euro una política económica común y vinculante tanto para los gobiernos nacionales como para el Banco Central Europeo, respetando las prerrogativas de cada institución.

Iniciaré la presentación describiendo la coordinación del marco institucional y de procedimiento en el área del euro. Sucesivamente describiré las evaluaciones de la literatura y las propuestas de reforma vinculadas. Finalmente, trataré 
de trazar un marco institucional y de procedimiento que refleje mejor las evaluaciones presentes en la literatura.

\section{EL MARCO INSTITUCIONAL}

La organización institucional de las actividades de coordinación atribuye un papel de liderazgo a la Comisión Europea, que inicia y completa los procedimientos de esta área. La Comisión se sostiene con la colaboración del Comité Económico-Financiero, coadyuvado por el Banco Central Europeo, para el análisis de la información disponible y la presentación de informes a las instituciones involucradas en la toma de decisiones. El poder formal de decisión es atribuido a un órgano político, el ECOFIN, que es la sección del Consejo de Ministros de la Unión Europea que se ocupa de cuestiones económicas y financieras. En las reuniones del ECOFIN participan, además de los representantes de la Comisión y del Banco Central Europeo, los ministros de economía y finanzas de todos los países de la Unión (también de aquellos que no adoptaron el euro). Para favorecer la eficacia de las actividades de coordinación en el área del euro, el Consejo de Europa creó en 1997 una estructura denominada Eurogrupo, en cuyas reuniones participan, además de los representantes de la Comisión y del Banco Central Europeo, sólo los ministros de economía y finanzas de los países que adoptaron el euro.

El eurogrupo debería desempeñar respecto al ECOFIN la misma función que el eurosistema desempeña en contra del Sistema Europeo de los Bancos Centrales. Sin embargo, el eurogrupo es, en la actualidad, sólo el lugar donde el Banco Central Europeo y los gobiernos nacionales de países que adoptaron el euro se reúnen para intercambiar opiniones e información en materia económica y financiera. No se atribuyó algún poder formal de toma de decisiones, poder que permaneció en el ECOFIN.

Este marco institucional no permite al Eurogrupo funcionar con la misma eficacia con la cual opera el Eurosistema en la política monetaria, y se cree que estas diferencias de poder llevan a los actores del proceso a asumir comportamientos no cooperativos, representan el principal problema de las actividades de coordinación entre las políticas monetaria y fiscal en el área del euro (véase Von Hagen y Mundschenk, 2003: 280, 293-294).

La falta de un lugar institucional, dentro del área, donde viene formalmente identificado aquello que se llama el policy-mix entre política fiscal y monetaria desincentiva la cooperación entre los diversos actores. La ausencia de decisiones comunes vinculantes para los gobiernos nacionales empuja al Banco Central 
Europeo a tutelar la propia autonomía para poderse contraponer eventualmente a aquello que considera la "propensión a generar déficit presupuestario" (sesgo deficitario) de los gobiernos nacionales. Por otra parte, la misma falta de decisiones comunes vinculadas, impulsa a los gobiernos nacionales a temer que las decisiones del Banco Central Europeo no tomen demasiado en cuenta los problemas de la economía del propio país y que las elecciones de política fiscal de los otros gobiernos sean tomadas persiguiendo los intereses nacionales en perjuicio de aquellos del área.

En conclusión, el sistema de incentivos que actualmente maneja las decisiones de los actores del proceso de coordinación de las políticas monetarias y fiscales en el área del euro, conduce a los gobiernos nacionales a tener posturas escasamente cooperativas entre ellos, y comportamientos volcados a neutralizar los eventuales efectos negativos de las políticas del Banco Central Europeo sobre la economía del propio país. Las políticas fiscales y monetarias en el área del euro son de esta manera conducidas, como muestran los análisis econométricos, no en forma complementaria, sino como "alternativas estratégicas": ésas tienden a neutralizarse recíprocamente, resultando ineficaces para el crecimiento económico del área.

\section{EL PROCEDIMIENTO}

Un documento del Banco Central Europeo, publicado con motivo del décimo aniversario de la fundación del área del euro, pone en evidencia que los Tratados de la Unión previenen tres diversos regímenes para la gestión de las políticas comunitarias (véase ЕсB, 2008a: 22). La gestión de la política monetaria fue enteramente transferida a la Comunidad, dando vida a la moneda y a la política monetaria única. Para la política fiscal los tratados prescriben un acercamiento basado en normas y procedimientos obligatorios (hard laws and procedures) con el fin de tener una coordinación eficaz entre las políticas nacionales y entre éstas y la política monetaria. Para las otras políticas, llamadas "estructurales", los tratados previenen formas "ligeras" (soft) de coordinación basadas en el diálogo a nivel comunitario y el apoyo mutuo entre los Estados miembros.

Los procedimientos previstos para la coordinación de las políticas monetarias y fiscales son numerosos. Un primer grupo introduce las mismas formas "ligeras" de coordinación existentes para las políticas "estructurales". La primera y más importante consiste en la elaboración de las Directrices generales para la política económica (Broad economic policy guidelines), propuestas cada año por la Comisión Europea con el apoyo del Comité Económico-Financiero y del Banco 
Central Europeo y aprobadas por el ECOFIN. Las "directrices" representan la base en la cual se ejerce a nivel comunitario la "vigilancia multilateral" sobre las políticas nacionales de parte de los órganos comunitarios y de los Estados miembros. En caso de incongruencia entre los contenidos de las "directrices" y las políticas nacionales, pueden ser activados otros procedimientos (los early warning y las peer pressures), que no tienen contenido coercitivo y son considerados de soft enforcement, en tanto intentan convencer con el diálogo a los Estados miembros de tener comportamientos conformes con los fines comunitarios.

Los tratados también establecen dos procedimientos: el "Pacto de Estabilidad y Crecimiento" y el "Procedimiento de déficit excesivo" diseñados para imponer a los Estados miembros, en caso de discrepancia entre las "directrices" y las políticas nacionales, la adopción de comportamientos coherentes con los objetivos comunitarios. Son éstos los procedimientos obligatorios (hard laws and procedures) que deberían consentir una gestión mayormente vinculante de la política fiscal en el área del euro.

El Pacto de estabilidad y crecimiento se basa en cuatro puntos y establece sanciones en caso de incumplimiento de las reglas.

El primer punto es la prohibición para las instituciones comunitarias de "rescatar" (bail out) a los gobiernos nacionales en graves dificultades financieras, es decir, en condiciones de no poder pagar su deuda.

El segundo es el límite impuesto a los gobiernos nacionales de no tener una deuda pública que supere el 60\% del Producto Interno Bruto (PIB).

El tercer punto es el límite impuesto a los gobiernos nacionales de no tener un déficit presupuestario real que supere el 3\% del PIB.

El cuarto es el requisito para los gobiernos nacionales de adoptar una política fiscal que tienda, en el mediano-largo plazo, al equilibrio presupuestal. En este caso, la restricción no es impuesta sobre el déficit presupuestario real, sino sobre la "tendencia" (cyclically adjusted).

El incumplimiento del Pacto de Estabilidad y Crecimiento puede poner en marcha el procedimiento de déficit excesivo, que puede imponer sanciones pecuniarias a los Estados miembros morosos.

Los gobiernos nacionales tuvieron serias dificultades para cumplir con las limitaciones del Pacto. Desde el nacimiento de la Unión Monetaria en 1999 hasta el 2006, las normas del Pacto fueron violadas por Portugal (2001, 2005 y 2006), Holanda (2003), Grecia (siempre), Francia (2002, 2003, 2004 y 2006), Alemania (de 2002 a 2005) e Italia (de 2003 a 2006). El elevado número de violaciones pone en evidencia la excesiva rigidez del Pacto y la necesidad de volverlo más flexible respecto de las necesidades reales de estabilización y crecimiento de las economías nacionales. 
La aplicación de los procedimientos de déficit excesivo también demostró ser difícil. Los casos de las violaciones de Francia y Alemania son los más emblemáticos. Los procedimientos de ejecución previstos y solicitados por la Comisón Europea fueron bloquedos por el ECOFIN en noviembre de 2003. La Comisión planteó el caso ante el Tribunal Europeo de Justicia, el cual anuló en 2004 la decisión del ECOFIn, estipulando sin ambargo, que la aplicación de los procedimientos de déficit excesivo no es automática y requiere siempre de una decisión adoptada por mayoría calificada del ECOFIN.

El complejo caso llevó, en marzo de 2005, a una reforma del Pacto de Estabilidad y Crecimiento y de los procedimientos de déficit excesivo. Tal reforma habría debido volver más flexibles, transparentes y vinculantes las normas sobre la coordinación de las políticas. De acuerdo con la literatura, sin embargo, ésta tuvo el efecto contrario, aumentando la ambigüedad de las reglas y reduciendo la aplicabilidad de las sanciones. La reforma, en lugar de fortalecer las normas y los procedimientos, como prescriben los tratados, redujo su eficacia favoreciendo la discrecionalidad nacional en la materia.

\section{EVALUACIONES CRÍTICAS Y PROPUESTAS DE CAMBIO}

Haciendo uso de algunas simplificaciones es posible subdividir la literatura sobre la coordinación de las políticas económicas en el área del euro en dos grupos de trabajos, uno que centra la atención en los cambios por introducir en los procedimientos, el otro que se centra en la necesidad de modificar también el marco institucional. Los dos grupos hacen referencia a posiciones diversas sobre el funcionamiento del sistema económico y atribuyen al término coordinación, dos significados diferentes.

El primer grupo de trabajos plantea que el término coordinación debe ser entendido como una actividad dirigida a regular el comportamiento de los gobiernos nacionales, garantizando independencia al Banco Central. Los trabajos de este primer grupo consideran necesario modificar los procedimientos para dar un contenido más transparente (menos ambiguo) a las restricciones impuestas a los gobiernos nacionales, conceder mayor flexibilidad a las políticas fiscales en función anti-cíclica, aumentar la "ejecución" (enforcement) de las autoridades competentes.

Para alcanzar estos objetivos los trabajos del primer grupo piden reducir el peso atribuido por los procedimientos existentes al valor real de la relación déficit público-PIB (punto 3 del Pacto), considerando oportuno concentrarse o sobre el valor de mediano-largo plazo (cyclically adjusted) de esta relación (punto 4 del 
Pacto), o sobre la relación deuda pública-Piв (punto 2 del Pacto), que mejor describe la sostenibilidad financiera de las políticas nacionales.

Fatàs y Mihov (2003) dan prioridad al valor de mediano-largo plazo de la relación déficit público-PIB, sosteniendo además que no es necesario indicar para eso un valor numérico específico. Según estos autores, los procedimientos "ligeros" de coordinación (la vigilancia multilateral, los warnings y las peer pressures) inciden en el comportamiento de los gobiernos nacionales más que los valores numéricos indicados por el Pacto de Estabilidad y Crecimiento.

Los análisis de Fatàs y Mihov subrayan la dificultad de hacer referencia a reglas fiscales no ambiguas. Los dos autores prefiren evitar la prescripción de valores numéricos precisos, mostrando tener más confianza en la eficacia de los procedimientos "ligeros" que en las ambigüedades relativas a la identificación teórica y contable de estos valores.

También Blanchard y Giavazzi (2004) privilegian el valor de mediano-largo plazo de la relación déficit público-pIB, afirmando que es "estúpido/insensato/ tonto" exigir que esta proporción deba ser cero. Hacer tender a cero el valor de esta proporción equivale a hacer tender a cero la proporción deuda pública-PIB, y es inútil e irracional, para los fines de la sostenibilidad financiera de las políticas fiscales, imponer que la segunda relación sea sujeta a tal restricción.

De acuerdo con Blanchard y Giavazzi los procedimientos de coordinación del área del euro deberían adoptar la llamada "regla de oro" (golden rule) de la política fiscal, la cual establece que tienda a cero el valor de mediano-largo plazo de la proporción entre el déficit público neto de los gastos de la cuenta de capital y el PIB.

Calmfors y Corsetti (2003) critican la golden rule porque introducen elementos de ambigüedad en la gestión de la política fiscal. Se ocupa de resolver el problema relativo a la tendencia del Pacto de Estabilidad y Crecimiento, a desincentivar el gasto público en la cuenta de capital, permitiendo así incrementar las potencialidades de crecimiento de la economía. La misma motivación que justifica la eliminación de la inversión pública por las limitaciones del Pacto pueden ser sin embargo, aplicadas para otras formas de gasto público, por ejemplo el de educación, que también tiene un impacto positivo sobre el potencial de crecimiento de la economía. Según Calmfors y Corsetti, las ambigüedades vinculadas a las motivaciones que aconsejan la adopción de la golden rule terminan por introducir elementos arbitrarios, sujetos a manipulaciones políticas, en la gestión de la política fiscal.

Como alternativa a la golden rule, Calmfors y Corsetti proponen recompensar a los gobiernos nacionales que mantienen una baja proporción entre la deuda pública y el PIB, elaborando una tabla numérica que fija, país por país, el 
cociente déficit-PIв que deben cumplir, relación identificada con base en el valor de la relación deuda pública-PIB que se registra en ese país.

Calmfors y Corsetti proponen además de reeforzar el poder de control previsto por los procedimientos de coordinación, transferir las decisiones en materia de la ECOFIn al Tribunal de Justicia Europea.

En la misma línea de dar mayor énfasis a la relación deuda pública-PIB se encuentran también Buiter y Grafe (2004), quienes proponen una regla, que llaman Permanent balance rule, derivada de las condiciones analíticas de sostenibilidad de la deuda pública. Tales condiciones asumen que el valor actual de los futuros superávit del presupuesto del Estado neto de los pagos de interés de la deuda pública, no debe ser menor que el valor actual de la misma deuda.

El objetivo de Buiter y Grafe es, una vez más, introducir flexibilidad en los procesos de coordinación. Según los autores, la Permanent balance rule permite superar los problemas de la golden rule, poniendo en el mismo plano el gasto público en la cuenta de capital y otras formas de gasto público que puedan influir positivamente sobre la potencialidad de crecimiento de la economía; tendría, por lo tanto, la cualidad de incentivar la adopción por parte de los gobiernos nacionales de todas las políticas que incrementan la potencialidad de crecimiento de la economía.

Frente a estos méritos, sin embargo, incluso la Permanent balance rule plantea cuestiones acerca de la arbitrariedad que estas reglas de política fiscal introducen en la gestión de los procedimientos de coordinación. La presencia de los valores esperados, no derivados de mediciones objetivas, en la fórmula que describe las condiciones de sostenibilidad de la deuda pública impone también tal regla a fuerte ambigüedad.

En conclusión, los intentos realizados hasta ahora para encontrar una norma fiscal que combine la flexibilidad y la transparencia parecen ser insuficientes. La literatura también evidencia que la norma del Pacto de Estabilidad y Crecimiento, la cual exige que el valor de mediano-largo plazo de la relación déficit público-PIB sea igual a cero está carente de fundamento analítico. Adoptar tal norma equivale a establecer que la relación deuda pública-PIB deba tender a cero, una imposición inútil para los fines de la sostenibilidad de la deuda. No existe motivo que haga creer que la sostenibilidad no pueda ser también garantizada por un proceso de estabilización hacia un valor positivo de la relación deuda pública-pIB. Un valor aceptable por los mercados no es definible en base a consideraciones teóricas; refleja el grado de confianza que los mercados muestran concretamente tener hacia las instituciones que gobiernan la economía de un país.

Aquellos que pertenecen al segundo grupo de trabajos describen la coordinación como un conjunto de actividades y de disposiciones institucionales con 
miras a identificar para toda el área del euro una política económica común y vinculante tanto para los gobiernos nacionales como para los organismos supranacionales, incluso el Banco Central Europeo, de conformidad con las prerrogativas de cada institución. Los autores aquí considerados sostienen que el actual marco institucional no contempla a los actores en el proceso de coordinación de los incentivos "justos", incitándolos a tener un comportamiento no cooperativo. Por tal motivo creen que sea necesario reformar los acuerdos institucionales y el sistema de incentivos para volver más eficaz la actividad de coordinación entre las políticas monetarias y fiscales. La posición más articulada es expresada por Wyplosz, que establece un paralelismo entre la evolución de las instituciones de la política monetaria y fiscal. Las críticas monetaristas de los ańos sesenta al modo de concebir la conducta de la política monetaria por parte de los keynesianos sugirieron el uso de reglas que guiarán las decisiones de las autoridades monetarias. Milton Friedman propuso la famosa "regla fija" que imponía una tasa constante de crecimiento de la oferta monetaria.

Los intentos de aplicar estas reglas, sin embargo, no han producido resultados satisfactorios y la política monetaria se volvió más eficaz desde que, abandonadas las reglas, se pasó al "diseño de las instituciones", a saber, a la organización de instituciones competentes dotadas de independencia y autonomía de acción. Los resultados de este nuevo enfoque fueron tan satisfactorios para inducir a los profesionales a usar la política monetaria como eje fundamental de la política económica.

Según Wyplosz (2002), las políticas fiscales están sufriendo, con cierto retardo, una evolución similar a la de la política monetaria. En los últimos ańos, todos los países adoptaron normas fiscales de diversa índole, pero ninguna produjo resultados satisfactorios. El fracaso de las reglas está convenciendo a los profesionales de que es necesario cambiar, también en este caso, en el terreno del "diseño institucional", proponiendo la creación de instituciones de política fiscal, dotadas técnicamente e independientes, al grado de evaluar, caso por caso, cuáles son las decisiones de política fiscal más idóneas para alcanzar los objetivos de estabilidad y crecimiento de las economías en las diversas situaciones. El lema que acompaña esta tendencia es competent judgement works better than rules.

Para el área del euro Wyplosz propone la creación de comités de política fiscal, análogos a los comités de política monetaria ya operantes. En su opinión, tales comités deben ser instituidos a nivel nacional, no a nivel europeo, no por razones de eficiencia, sino para facilitar su introducción disminuyendo la resistencia de los gobiernos nacionales para ceder soberanía en materia fiscal.

$\mathrm{Al}$ igual que en la política monetaria, los gobiernos deberían "delegar" a los comités de política fiscal la búsqueda de un objetivo bien definido. El objetivo 
delegado a las autoridades monetarias independientes es el de la estabilidad de precios. El objetivo para delegar a los comités de política fiscal, según Wyplosz, no puede referirse a la composición del gasto público y la estructura tributaria. Las decisiones sobre estos puntos tienen un contenido político inmediato, en cuanto inciden directamente sobre la distribución de los beneficios y de los costos, transfiriéndolos de algunos grupos sociales a otros. Ésas por lo tanto, deben ser tomadas por organismos políticos democráticamente electos. Las decisiones sobre la dimensión del déficit público pueden, sin embargo, ser delegadas. También estas decisiones tienen efectos redistributivos, pero éstas, como sucede para aquellas derivadas de las decisiones de política monetaria sobre las tasas de inflación y de interés, se producen a través de la operación compleja de los mecanismos del mercado y no como consecuencia inmediata de las decisiones tomadas por las autoridades competentes.

Los comités de política fiscal deberían por lo tanto, tomar decisiones sobre el valor de la relación déficit público-PIв más apropiado para cada país en las diversas situaciones cíclicas y estructurales por las que atraviesa la economía. A través de tales decisiones los comités deberían perseguir, por cuenta de los organismos democráticamente electos, el objetivo de la sostenibilidad de la política fiscal, es decir, el financiamiento regular de la deuda pública por parte de los mercados financieros.

La comparación entre la política monetaria y la fiscal delineada por Wyplosz tiene, también en este caso, sentido. A las autoridades monetarias independientes los gobiernos delegan el objetivo de la estabilidad de precios que deben perseguirse con el instrumento de las variaciones de la tasa de interés. A los técnicos de la política fiscal los gobiernos les delegan el objetivo de la sostenibilidad financiera que debe buscarse con el instrumento de las variaciones de la relación déficit público-PIB.

Los análisis de Wyplosz representan una contribución central para la comprensión y resolución de los problemas de coordinación. Éstos esbozan una línea evolutiva de la literatura especializada que difícilmente puede ser descuidada por quien se mide con las propuestas de reforma en materia.

Un punto de sus posiciones, sin embargo, puede causar alguna confusión. Wyplosz propone que los comités de política fiscal sean instituidos a nivel nacional para atenuar la resistencia de los gobiernos a ceder soberanía en materia fiscal. Tal propuesta tiende a subestimar la relevancia de las dimensiones supranacionales y la necesidad evidenciada por los análisis sobre los incentivos "justos" para la eficacia de las actividades de coordinación, para identificar a nivel de toda el área del euro una policy mix fiscal y monetaria vinculante para todos los actores del proceso. En sus trabajos, Wyplosz se centra sobre estos problemas, no 
ofrece ninguna justificación del por qué, en su opinión, los gobiernos nacionales deberían estar más dispuestos a delegar las decisiones sobre el tamaño del déficit público a un organismo independiente a nivel nacional, más que a un organismo a nivel supranacional, por ejemplo a una institución similar al Eurogrupo, donde podrían ser directamente representados interactuando con el trabajo del personal técnico.

Una posición análoga fue presentada por Pisani-Ferry (2002) que insiste más que Wyplosz sobre la necesidad de no descuidar el nivel supranacional y la identificación de una policy mix para el área completa del euro. Sus propuestas, sin embargo, también preocupadas por la resistencia de los actores del proceso de coordinación, no resultan plenamente coherentes con el contenido de sus evaluaciones.

Para dar relevancia al nivel supranacional y a la identificación de una policy mix para el área completa del euro, Pisani-Ferry sugiere transformar al Eurogrupo en la Agencia Fiscal del área del Euro. Temiendo, sin embargo, la oposición de los gobiernos a la cesión de soberanía fiscal a los organismos supranacionales, propone que las decisiones de este organismo no sean limitadas, pero se basen en la capacidad de los procedimientos "ligeros" de coordinación para convencer a los gobiernos de adoptarlas. Además, temiendo la resistencia del Banco Central a aceptar una reducción de la propia autonomía de acción, Pisani-Ferry propone que el Banco Central Europeo, no sea limitado por las decisiones del reformado Eurogrupo.

Estas elecciones de Pisani-Ferry quitan fuerza a sus propuestas, ya que terminan descuidando las conclusiones de la literatura sobre el rol que la ausencia de decisiones vinculantes tiene en el fomentar comportamientos no cooperativos por parte de los gobiernos nacionales. Además indican una limitada profundización del problema de la autonomía de los bancos centrales.

La literatura sobre la materia, distingue diversas formas de independencia que pueden ser atribuidas a un Banco Central (véase Panico y Rizza, 2004). Define "independencia técnica" como aquella relativa a la elección de los instrumentos más idóneos en las diversas situaciones. En general, los bancos centrales de todo el mundo disfrutan de la posibilidad de decidir autónomamente, con base en su competencia profesional, qué instrumentos usar y en qué medida usarlos. Esta forma de independencia no es la única identificada por la literatura, que habla entre otras cosas también de la "independencia sobre los objetivos" y de "independencia sobre las prioridades", relativas a la posibilidad ofrecida a un Banco Central para fijar autónomamente el nivel a perseguir de las variablesobjetivo (por ejemplo, el nivel de la tasa de inflación que debe ser perseguido) y el grado de prioridad por atribuir al objetivo principal (la tasa de inflación) 
en comparación con los demás objetivos de la política monetaria. Tales formas de independencia son atribuidas sólo al Banco Central Europeo. En otras economías, la responsabilidad de fijar el nivel de las variables-objetivo es atribuida a los organismos democráticamente electos (Parlamento y Gobierno). La asignación de estas formas de independencia del Banco Central Europeo es, por lo tanto, un hecho anómalo, que a la larga puede deslegitimar y resultar peligroso para el mismo Banco Central. Permaneciendo la garantía de la independencia técnica, la participación del Banco Central Europeo en las actividades del reformado Eurogrupo y la adhesión por parte de las mismas decisiones que en aquella sede se tomen sobre la coordinación de las políticas económicas, no afecten al Banco Central y la política monetaria. Por el contrario, esta solución puede allanar el camino para un nuevo equilibrio entre las prerrogativas del Banco Central y de los otros órganos de gobierno de la economía, hacia una actitud mayormente cooperativa de los diferentes actores del proceso de coordinación de las políticas fiscales y monetarias y, por lo tanto, a una mayor eficacia de la misma coordinación.

\section{CONCLUSIONES}

¿Qué podemos aprender de esta revisión de la literatura? Resumo a continuación tres puntos principales con los que debe medir quien quiere enfrentar el problema de las actividades de coordinación en el área del euro en términos propositivos:

1. Debido a la incertidumbre causada por la falta de decisiones vinculantes, el sistema de incentivos previsto por el actual marco institucional y de procedimiento no induce a los actores del proceso de coordinación a tener comportamientos cooperativos.

2. El diseño de los Tratados, que tenía previsto para la política fiscal una actividad de coordinación basada sobre hard laws and procedures, fue rechazado: los procedimientos que debían garantizar la realización de este proyecto resultaron inadecuados y se volvieron aún más después de la reforma de marzo de 2005.

3. Como lo fue para la política monetaria, también para la política fiscal la adopción de reglas se muestra insatisfactoria y los expertos están de acuerdo en que, también en este caso, es necesario moverse sobre el terreno del "diseño institucional”, proponiendo la creación de instituciones técnicamente equipadas y dotadas de un suficiente grado de independencia, capaces de determinar, caso por caso, cuáles son las decisiones de política fiscal más 
adecuadas para alcanzar los objetivos fijados por los organismos democráticamente electos.

La propuesta de Pisani-Ferry de transformar el Eurogrupo en la Agencia Tributaria del Área del Euro es el punto de partida más apropiado para el diseño de una reorganización de las actividades de coordinación de conformidad con los tres puntos arriba señalados. Además de hacer realizar al Eurogrupo una función análoga a aquella que el Eurosistema desarrolla en relación con el Sistema Europeo de Bancos Centrales, la transformación de este organismo en Agencia Tributaria hace posible identificar una policy mix fiscal y monetaria, vinculante para los gobiernos nacionales y el Banco Central Europeo y la eliminación del principal problema actualmente presente en el sistema de incentivos.

EI reformado Eurogrupo debería ser dotado de una competente estructura técnica y, como los Comités de política monetaria, presentar en su interior un satisfactorio equilibrio entre el papel de los componentes técnicos y el de los componentes políticos.

El proceso de reforma que se va esbozando debería garantizar al Banco Central el respeto de su "independencia técnica". Las decisiones del reformado Eurogrupo, tomadas con la participación directa del Banco Central Europeo, deberían indicar las limitaciones impuestas a las políticas fiscales de los gobiernos nacionales y la manera en la que se decidió complementar las opciones de política fiscal y monetaria, estableciendo así el grado de prioridad atribuido a los diversos objetivos de la política económica. La aplicación práctica de la parte monetaria de esta policy mix debe dejarse al criterio de las autoridades monetarias, las cuales deberán sentirse garantizadas también en la contundencia de las limitaciones impuestas a los gobiernos nacionales, así como estos últimos deben sentirse seguros de que todas las partes interesadas respetarán las decisiones adoptadas en el interior del Eurogrupo.

Con el fin de garantizar estas condiciones parece oportuno introducir cambios también en los procedimientos relativos a los beneficios y las sanciones. En primer lugar, parece oportuno revisar el Pacto de Estabilidad y Crecimiento manteniendo las prohibiciones de bail out y de tener una deuda pública superior al $60 \%$ del pib y cambiando el requisito, inútil e irracional, para los gobiernos nacionales de adoptar una política fiscal que tenga, en el mediano-largo plazo, el presupuesto del estado en equilibrio. Por último, para asegurar la coherencia entre las reglas del pacto y los documentos del Banco Central Europeo, el valor a mediano-largo plazo del déficit público-PIB, que representa el valor de referencia de la conducta de la política fiscal debe estar comprendido entre el 2.4 y el 2.7\%. Una inmediata elaboración de análisis matemáticos muestra que estas pruebas 
garantizan la convergencia de la relación deuda pública-PIB al 60\% cuando la tasa de inflación perseguida como objetivo de la política monetaria es del $2 \%$ y la tasa de crecimiento real de la economía, según lo indicado por el Banco Central Europeo como realista en el mediano-largo plazo, está comprendida entre el 2 y el 2.5\%. El reformado Eurogrupo establecerá año con año, según las exigencias cíclicas y estructurales de las economías individuales, si y en cuánto el valor real de la relación déficit público-PIв de cada país podrá diferir del valor de referencia del mediano-largo plazo. En caso de relevantes y comprobados problemas estructurales (por ejemplo, la pérdida persistente de competitividad internacional) el reformado Eurogrupo puede conceder a una economía restar del cálculo de la relación del déficit público-PIB el gasto de las intervenciones acordadas para su solución y, como una forma adicional de incentivación para el respeto de las reglas del Pacto, puede disponer que dichos gastos sean financiados con el apoyo de las instituciones europeas o mediante la emisión de bonos.

Para dar mayor fuerza y legitimidad a las decisiones del reformado Eurogrupo, los parlamentos y los gobiernos nacionales deberían también organizar regularmente discusiones públicas sobre su situación económica, financiera y fiscal.

Por último, para reforzar las garantías de respeto a las decisiones tomadas, los procedimientos de ejecución deberían ser fortalecidos, transfiriendo las facultades del Ecofin al Tribunal Europeo de Justicia, reduciendo los tiempos para la imposición de las sanciones.

\section{REFERENCIAS BIBLIOGRÁFICAS}

Beestma R., and Debrun X., Implementing the Stability and Growth Pact: Enforcement and Procedural Flexibility, есв Working Paper, num. 433, January, 2005.

Blanchard O. J. and Giavazzi F., Improving the SGP Through a Proper Accounting of Public Investment, CEPR Discussion Paper Series, num. 4220, 2004.

Buiter W.H. and Grafe C., "Patching up the Pact: Suggestions for Enhancing Fiscal Sustainability and Macroeconomic Stability in an Enlarged European Union", Economics of Transition, 12(1), 67-102, 2004.

Buti M., Eijffinger S. and Franco D., Revisiting the Stability and Growth Pact: Grand Design or Internal Adjustment?, Mimeo, 2002. http://www.cepii.fr/ anglaisgraph/communications/pdf/2002/211102/franco.pdf

Calmfors L., "What remains of the Stability Pact and what next?", Swedish Institute for European Policy Studies, Report num. 8, November, 2005. 
Calmfors L. and Corsetti G., How to Reform Europe's Fiscal Policy Framework, mimeo, 2003.

http://rincewind.iies.su.se/-lcalmfor/wejjan16calmfors.pdf

есв (European Central Bank), Monthly Bulletin: Tenth Anniversary of the ECB, http://www.ecb.int, 2008.

Fatàs A. and Mihov I., "On Constraining Fiscal Policy Discretion in EMu", Oxford Review of Economic Policy, 19 (1), 112-31, 2003.

Panico C. and Rizza M.O., "Central Bank Independence and Democracy: a Historical Perspective", in Arena R. and Salvadori N., eds., Money, Credit and the Role of the State: Essays in Honour of Augusto Graziani, Ashegate, London, 447-67, 2004.

Panico C. and Vázquez Suárez M., "Policy Coordination in the Euroarea", Studi Economici, 96(3), 5-31, 2008.

Pisani-Ferry J., Fiscal Discipline and Policy Coordination in the Eurozone: Assessment and Proposals, mimeo, 2002. http://www.pisani-ferry.net/base/ re02-gea-discipline-vmai.pdf

Von Hagen J. and Mundschenk S., "Fiscal and monetary policy coordination in EMU", International Journal of Finance and Economics, 8, 279-95, 2003.

Wyplosz C., Economic Policy Coordination in EMU: Strategies and Institutions, mimeo, 1999.

http://hei.unige.ch/ - wyplosz/cw_bonn_final.pDF

Wyplosz C., Fiscal discipline in EMU: rules or institutions?, mimeo, 2002. http:// hei.unige.ch/ - wyplosz/gea_0402.pdf 